\title{
Photoisomerization Action Spectrum of Retinal Protonated Schiff Base in the Gas
} Phase

\author{
N. J. A. Coughlan, ${ }^{1}$ K. J. Catani, ${ }^{1}$ B. D. Adamson, ${ }^{1}$ U. Wille, ${ }^{1}$ and E. J. Bieske ${ }^{1,}$ a)
} School of Chemistry, The University of Melbourne, Victoria, Australia 3010

(Dated: 16 September 2014)

The photophysical behaviour of the isolated retinal protonated n-butylamine Schiff base (RPSB) is investigated in the gas phase using a combination of ion mobility spectrometry and laser spectroscopy. The RPSB cations are introduced by electrospray ionisation into an ion mobility mass spectrometer where they are exposed to tuneable laser radiation in the region of the $S_{1} \leftarrow S_{0}$ transition (420-680 nm range). Four peaks are observed in the arrival time distribution of the RPSB ions. On the basis of predicted collision cross sections with nitrogen gas, the dominant peak is assigned to the all-trans isomer, whereas the subsidiary peaks are assigned to various single, double and triple cis geometric isomers. RPSB ions that absorb laser radiation undergo photoisomerization, leading to a detectable change in their drift speed. By monitoring the photoisomer signal as a function of laser wavelength an action spectrum, extending from 480 to $660 \mathrm{~nm}$ with a clear peak at $615 \pm 5 \mathrm{~nm}$, is obtained. The photoisomerization action spectrum is related to the absorption spectrum of isolated retinal RPSB molecules and should help benchmark future electronic structure calculations.

Keywords: retinal, electrospray ionization, ion mobility spectrometry, photoisomerization, laser spectroscopy

a)Electronic mail: evanjb@unimelb.edu.au 


\section{INTRODUCTION}

Retinal and its analogues play key functions in vision and photosynthesis, transforming photon energy into the structural reorganisation of proteins with prodigious efficiency. For example, the photoisomerization of the protonated retinal Schiff base (RPSB) plays a role in rhodopsin, the primary unit for vision, with the photoisomerization from 11cis to the trans configuration being the primary event in the visual transduction process. In bacteriorhodopsin and halorhodopsin, which function as light activated proton and chloride pumps, respectively, the trans form of RPSB is photoisomerised to the 13-cis form. Remarkably, the absorption maximum of retinal can be tuned over a broad wavelength range by changing the RPSB configuration, protein environment and proximity of the counter ion, and occurs at $489 \mathrm{~nm}$ in rhodopsin, $568 \mathrm{~nm}$ in bacteriorhodopsin, $578 \mathrm{~nm}$ in halorhodopsin and $446 \mathrm{~nm}$ in ethanol solution. ${ }^{1,2}$ For retinal embedded in a protein, photoisomerization occurs on a 450-500 fs timescale, ${ }^{3}$ has a high quantum yield (0.64), and proceeds with extraordinary specificity for a particular product photo isomer. ${ }^{4}$ Understanding how the wavelength of the retinal absorption is governed by conformation and interactions with the surrounding protein and counter ion has long stood as a challenge for experimental and theoretical biophysicists. ${ }^{5}$ The current degree of control is highlighted by the recent encapsulation of all-trans RPSB in selectively modified versions of the human cellular retinol binding protein II (hCRBPII), ${ }^{6}$ in which electrostatic interactions controlling the electronic properties of the RPSB were modulated by introducing point mutations in the protein, thereby allowing tuning of the absorption maximum from 425 to $644 \mathrm{~nm}$. In other recent studies, control over the excited state lifetime, the photoisomerization pathway and photoisomerization yield for RPSB in solution has been achieved chemically through the introduction of polarisable backbone substituents. 7,8

Although intermolecular interactions between the protein and the retinal molecule are capable of shifting the absorption maximum by several hundred $\mathrm{nm}$, there is less certainty about the role of the inherent retinal conformation on the absorption wavelength. Indeed, within a protein environment it is difficult to disentangle intermolecular and intramolecular effects. In principle, the role of conformation can be elucidated more clearly by removing the protein scaffold and investigating the isolated retinal molecule. A common target for theoretical ${ }^{5,9}$ and experimental studies ${ }^{10-15}$ is the retinal protonated 
n-butylamine Schiff base (Figure 1), which includes the n-butylamine group that usually tethers the retinal moiety to the surrounding native protein.

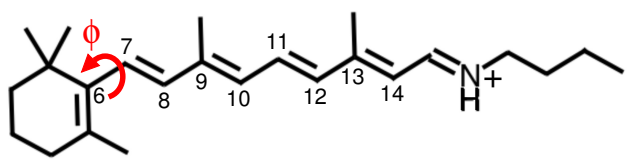

FIG. 1. The all-trans retinal protonated Schiff base. DFT calculations by Valsson et al. at the M062X/cc-pVDZ level predict that the isomers are 6s-cis(-) with $\phi=-38.8^{\circ} \Delta E=0 \mathrm{~kJ} / \mathrm{mol}, 6 \mathrm{~s}-$ trans with $\phi=-170.1^{\circ} \Delta E=+0.5 \mathrm{~kJ} / \mathrm{mol}$, 6s-cis $(+)$ with $\phi=+41.8^{\circ} \Delta E=+1.4 \mathrm{~kJ} / \mathrm{mol} .^{9}$

Generating experimental spectroscopic data for isolated RPSB ions in the gas phase against which different computational approaches can be calibrated requires deploying a sufficiently sensitive spectroscopic technique for ultra-low concentrations, and preferably ensuring that the probed molecules have a single configuration so that an unambiguous connection can be established between the configuration and absorption spectrum. In pioneering studies, Andersen and coworkers probed the electronic transitions of nbutylamine RPSB ions in an ion storage ring by photodissociating them with a pulsed, tunable laser beam while monitoring neutral photofragments. ${ }^{10,11,13,14}$ The most recent photodissociation (PD) spectrum, ${ }^{13}$ which differs somewhat from previously reported versions, ${ }^{10,11}$ exhibits a broad peak with a flat-topped maximum extending from $610 \mathrm{~nm}$ down to $530 \mathrm{~nm} .{ }^{13}$ The PD spectrum was interpreted as reflecting overlapping contributions from 6s-trans and 6s-cis configurations of RPSB (see Fig. 1) with peak absorptions at 610 and $530 \mathrm{~nm}$, respectively. ${ }^{13}$

Here we investigate the electronic transitions and photoisomerization of RPSB using an alternative form of action spectroscopy, PhotoISomerization Action (PISA) spectroscopy. ${ }^{16,17}$ The approach is a modification of ion mobility spectrometry (IMS), whereby ions drifting under the influence of an electric field through a buffer gas (usually helium or nitrogen) are separated spatially and temporally according to their collision cross section, which in turn depends on the molecular conformation. In the current work, we monitor photoisomerization from all-trans RPSB to different cis versions of RPSB as the ions drift under the influence of an electric field through a cell filled with $\mathrm{N}_{2}$ buffer gas. Generally, the cis forms of RPSB are more compact than the trans form and travel through the drift cell 
more rapidly. We have previously deployed the approach to investigate the electronic absorptions and photoisomerization of the carbocyanine dye cations $\mathrm{HICC}^{+}$and $\mathrm{DTC}^{+}$in the gas phase. ${ }^{16,17}$

Our approach has several potential advantages for investigating the photophysical behaviour of RPSB. First, even without laser excitation, IMS provides information on the different RPSB isomers generated by the electrospray ionisation source. Second, by monitoring the intensity of the photo isomer peaks or depletion of the parent peak as a function of laser wavelength, one can generate an action spectrum that is related to the absorption spectrum convoluted with isomerization probability (which may depend on wavelength). For RPSB, the action spectrum obtained in this way complements the PD action spectra obtained by monitoring photo fragments. ${ }^{10,11,13,14}$ Trans-cis isomerization barriers for RPSB are typically around $100 \mathrm{~kJ} / \mathrm{mol}$, much less than bond dissociation energies $(300-400 \mathrm{~kJ} / \mathrm{mol})$, ensuring that the entire thermal population potentially contributes to the PISA spectrum.

\section{METHODS}

The experimental approach involves irradiating protonated RPSB in a custom built ion mobility apparatus. ${ }^{16,17}$ The experimental layout is shown in Figure 2. RPSB cations were electrosprayed from a $10^{-5} \mathrm{M}$ solution of trans RPSB in 1:1 methanol: $\mathrm{H}_{2} \mathrm{O}$ with a few drops of acetic acid (electrospray voltage $3 \mathrm{kV}$, flow rate $\sim 5 \mu \mathrm{L} / \mathrm{min}$ ). The ions passed through a heated desolvation capillary $(T=500 \mathrm{~K})$ and were accumulated in an ion funnel before being launched in a $150 \mu$ s pulse into a $0.9 \mathrm{~m}$ drift tube filled with $\mathrm{N}_{2}$ buffer gas ( $\mathrm{P}=14$ torr). The electric field in the drift tube $(44 \mathrm{~V} / \mathrm{cm})$ was sustained by a series of 90 ring electrodes. At the end of the drift tube, the ions were gathered radially by a second ion funnel before passing through a $0.3 \mathrm{~mm}$ orifice into an octopole ion guide from which they exited through a $3 \mathrm{~mm}$ orifice into a quadrupole mass filter (QMF) set to $m / z=340$ amu. Ions exiting the QMF were sensed by a channeltron detector connected to a discriminator and a multichannel scaler (MCS). The apparatus was run at $20 \mathrm{~Hz}$ and the ions' arrival time distribution (ATD) built up as a histogram of ion counts versus time. The mobility resolution for the RPSB ions was typically $t_{d} / \Delta t_{d}=60$, which can be compared to a diffusion-limited resolution under the prevailing conditions of $120 .{ }^{16}$ Under typical 


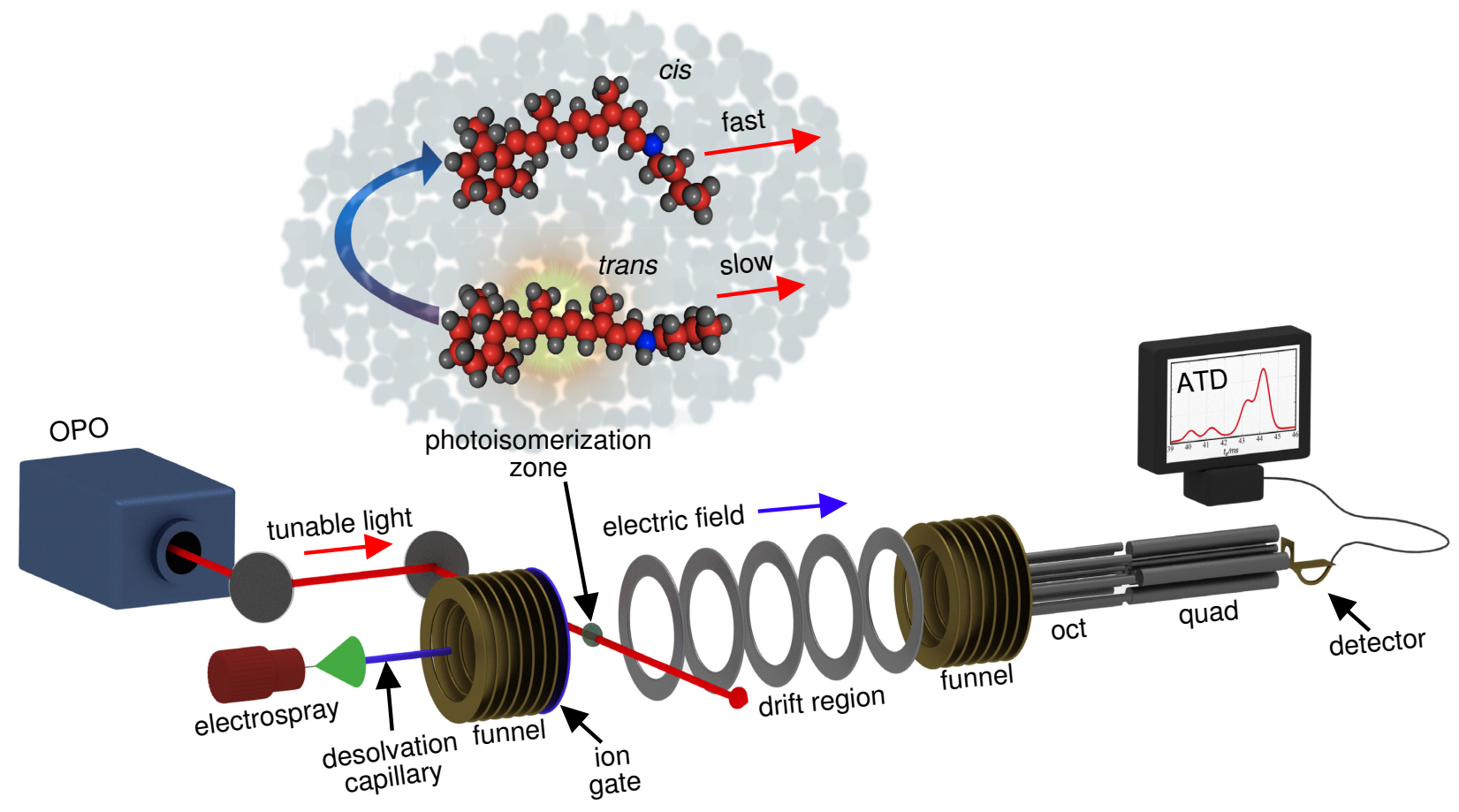

FIG. 2. Schematic illustration of the gas-phase RPSB photoisomerization experiment. Electrosprayed RPSB ions are collected by an ion funnel and injected into a $0.9 \mathrm{~m}$ drift tube filled with $\mathrm{N}_{2}$ buffer gas where they are exposed to tuneable visible radiation. The ions then drift under the influence of an electric field through the buffer gas before being collected by a second ion funnel, and passed through an octopole and a quadrupole mass filter. Photoisomerization changes the ions' collision cross section and speed, such that they arrive at the ion detector earlier or later than the parent ions.

operating conditions with a drift field of $44 \mathrm{~V} / \mathrm{cm}, \mathrm{N}_{2}$ buffer gas pressure and temperature of 14 torr and $295 \mathrm{~K}$, respectively, the effective temperature of the ions is predicted to be $T_{\text {eff }}=303 \mathrm{~K} \cdot{ }^{18}$

For the photoisomerization studies, alternate ion packets were irradiated immediately after exiting the first ion funnel with the $10 \mathrm{~Hz}$ output of a pulsed, tunable optical parametric oscillator (OPO, $\lambda=410-710 \mathrm{~nm}, 10 \mathrm{~ns}$ pulse width, $5 \mathrm{~cm}^{-1}$ bandwidth, $5-10 \mathrm{~mJ} / \mathrm{pulse}$, $\sim 5 \mathrm{~mm}$ beam diameter). The laser-induced change was recorded as the difference between laser-on and laser-off ATD histograms. Typically, the energy was 5-10 mJ/pulse over the RPSB absorption band and had a cross section of $\sim 5 \mathrm{~cm}^{2}$, corresponding to energy fluxes 
of $1-2 \mathrm{~mJ} / \mathrm{cm}^{2} /$ pulse. The photoisomerization signal was normalised by photon number. All RPSB isomers present in the ion packet are simultaneously exposed to light at the beginning of the drift region and are therefore potentially depleted or augmented by the light pulse.

At the beam intensities used in this study photoisomerization was the dominant process with the number of RPSB ions undergoing photoisomerization at least 100 times greater than the number photodissociating (see Section III A). However, if we increased the laser beam intensity by an order of magnitude (to $20-30 \mathrm{~mJ} / \mathrm{cm}^{2}$ at $532 \mathrm{~nm}$ ), we clearly observed $m / z=248$ amu photofragments, as found in previous fast ion beam studies. ${ }^{15}$ These photofragments have the same drift mobility as background $m / z=248$ amu ions generated directly by the ion source. We believe that in our experiment the $m / z=248$ amu photofragments at these higher intensities (which are well above those used for the photoisomerization investigations described in this paper) arise from multi-photon excitation of the RPSB.

The all-trans RPSB was synthesized under exclusion of light in an Ar atmosphere according to previous methods. ${ }^{19}$ Briefly, all-trans retinal (Sigma Aldrich) was added to an equimolar amount of $\mathrm{n}$-butylamine (Sigma Aldrich) in dry $\mathrm{CHCl}_{3}$. The mixture was heated to reflux conditions until 1H NMR showed complete disappearance of the carbonyl signal at approximately $10 \mathrm{ppm}$ ( $\mathrm{ca} 1 \mathrm{hr}$ ) following which the product was collected by evaporation of the solvent under reduced pressure. The isomeric purity of the product was established by HPLC.

\section{RESULTS AND DISCUSSION}

\section{A. Arrival time distribution of electrosprayed RPSB cations}

The arrival time distribution (ATD) for RPSB ions $(m / z=340 \mathrm{amu})$ without laser radiation is shown in Figure 3a. Although the electrosprayed sample was prepared as trans RPSB, the ion mobility data provide clear evidence that at least 4 different isomers with $m / z=340 \mathrm{amu}$ are present in the drifting ion population (Peaks A-D). Peak arrival times, collision cross sections, and tentative assignments are given in Table I. We discuss the peak assignments later in Section IV, remarking here only that the dominant, slow 


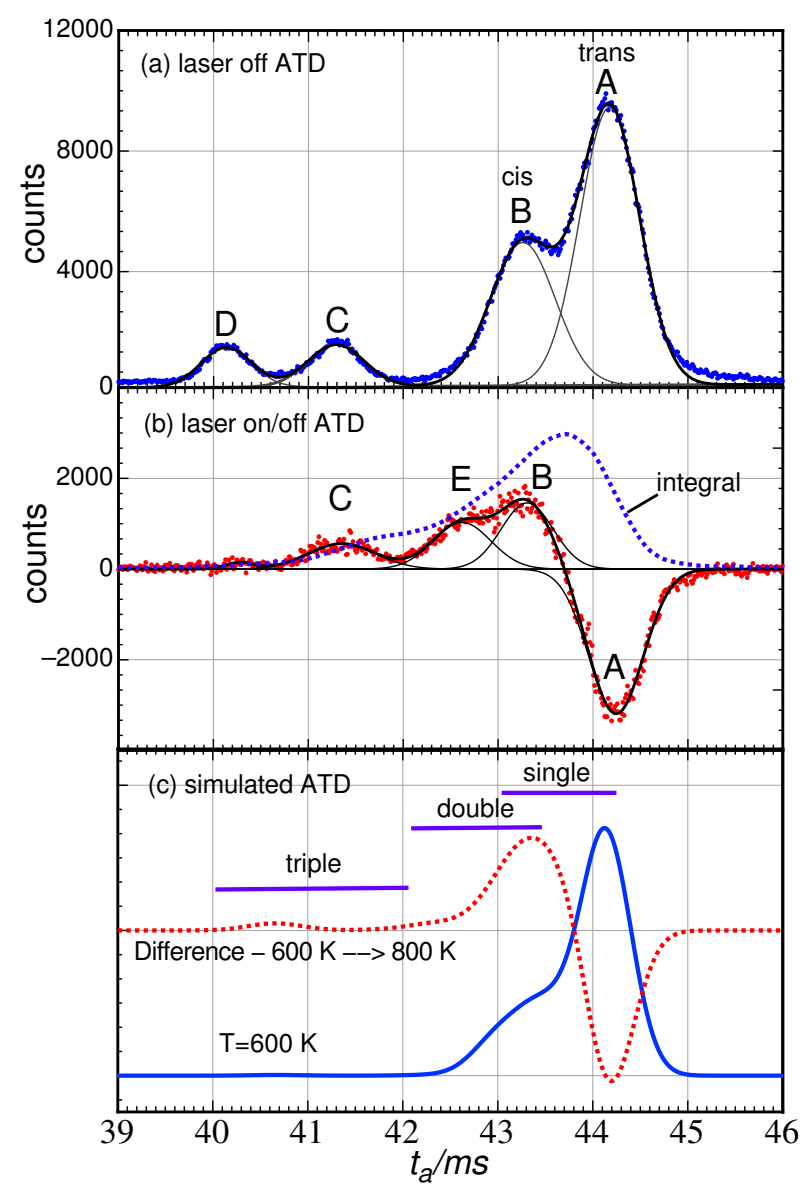

FIG. 3. (a) Arrival time distribution for electrosprayed RPSB. (b) Difference between laser-off and laser-on ATDs. Peaks have each been fitted with Gaussian functions ( $\mathrm{fwhm}=0.7 \mathrm{~ms}$ ) as indicated by the solid lines. The dotted line is proportional to the integral of the laser on/off difference signal with respect to arrival time. (c) Simulated ATD assuming a Boltzmann distribution of isomers at $\mathrm{T}=600 \mathrm{~K}$ (solid line), and difference between ATDs at $T=800 \mathrm{~K}$ and $600 \mathrm{~K}$ (dotted line). Ranges for predicted arrival times for single, double and triple cis isomers are indicated by horizontal lines.

peak (peak A) is almost certainly due to the all-trans RPSB isomer and that the earlier peaks arise from either geometric RPSB isomers, RPSB protomers (structures in which the proton is localised on a C atom), or possibly cyclic forms of RPSB. These other isomers are presumably formed either in the electrospray process or during passage through the desolvation capillary where the temperature is $\sim 500 \mathrm{~K}$.

The effect of laser excitation at $590 \mathrm{~nm}$ on the drifting RPSB population is illustrated in Figure $3 b$ where the difference between the laser-on and laser-off ATDs is plotted. There is 
TABLE I. Arrival times $\left(t_{a}\right)$, collision cross sections $\left(\sigma_{\text {meas }}\right)$, and assignments for experimental ATD peaks shown in Figure 3.

\begin{tabular}{ccccc}
\hline \hline Peak & $t_{a}$ & $\sigma_{\text {meas }}$ & assignment & formation \\
& ms & $\AA^{2}$ & & \\
\hline A & 44.15 & 227.9 & trans, 8c, 12c, 13c & thermal/photo \\
B & 43.25 & 222.6 & $9 \mathrm{c}$ & thermal/photo \\
C & 41.30 & 212.5 & triple-cis, cyclic & thermal/photo \\
D & 40.10 & 206.3 & triple-cis, cyclic & thermal \\
E & 42.60 & 219.2 & double-cis/triple-cis & photo \\
\hline
\end{tabular}

an obvious depletion of the lowest mobility species (peak A) accompanied by formation of the isomer(s) associated with peaks B and C. Peak D is enhanced only very slightly by laser irradiation. Significantly, a new photo-induced isomer peak appears at $42.60 \mathrm{~ms}$ (peak E), which is in the region expected for double-cis or triple-cis isomers (see Section IV). The isomer(s) responsible for peak $\mathrm{E}$ is (are) either formed by a direct photochemical transformation or requires more energy than is available thermally. Crucially, the depletion of peak A is completely balanced by enhancements of peaks B, C and E, as demonstrated by the fact that the integral of the difference ATD signal with respect to arrival time (dotted curve in Figure $3 \mathrm{~b}$ ) returns to zero (to within $1 \%$ ) by $t_{a}=45.5 \mathrm{~ms}$. Given that the ATD was recorded with the quadrupole mass filter set to $m / z=340 \mathrm{amu}$, this is compelling evidence that photoisomerization is the only significant photo-process and that photodissociation and other loss mechanisms are negligible under the operative experimental conditions.

\section{B. Photoisomerization Action Spectrum}

We turn now to a discussion of the wavelength dependence for the RPSB photoisomerization. The difference between the laser-on and laser-off ATDs as a function of OPO wavelength is presented as a two-dimensional plot in Figure 4 where it can be seen that the wavelength dependence for the depletion of the slowest ions (peak A), which is assigned to the all-trans isomer of RPSB, is very similar to the wavelength dependence for the 


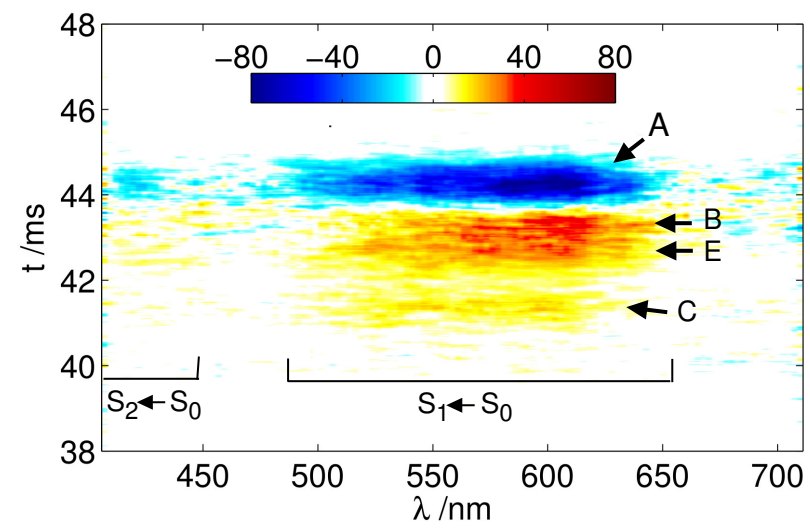

FIG. 4. Difference between laser-on and laser-off ATDs as a function of wavelength (x-axis) and ion arrival time (y-axis) with the mass spectrometer set to $m / z=342 \mathrm{amu}$. The blue region corresponds to a laser-induced depletion of trans RPSB ions (Peak A in Fig. 3), and the red/yellow regions to augmentation of faster, more compact isomeric structures (peaks B, C, E in Fig. 3).

growth of the photo isomers (peaks B, C \& E). The wavelength dependence of the photo activity is more apparent in Figure 5 where the depletion of the all-trans RPSB signal (Peak A) is plotted as a function of wavelength. The observed band, which corresponds to the $\mathrm{S}_{1} \leftarrow \mathrm{S}_{0}$ transition, ranges from 440 to $660 \mathrm{~nm}$ with a distinct maximum at $615 \pm 5 \mathrm{~nm}$. The photo-enhancements of peaks B, C and E are also plotted as function of wavelength in Figure 5 and display a similar wavelength dependence, although, the maximum of the curve for peak $C$ is perhaps slightly blue-shifted by $10 \mathrm{~nm}$ to $605 \mathrm{~nm}$. The gas-phase PISA absorption band is red-shifted by $170 \mathrm{~nm}$ from the peak of the $S_{1} \leftarrow S_{0}$ band for RPSB in acetonitrile $\left(\lambda_{\max }=445 \mathrm{~nm}\right)$. There is also evidence for photoisomerization below $450 \mathrm{~nm}$ in the spectral region associated with the onset of the $S_{2} \leftarrow S_{0}$ transition. ${ }^{11}$

Notably, the shape of the PISA $S_{1} \leftarrow S_{0}$ band differs from the most recently reported photodissociation (PD) spectrum of RPSB, which exhibits a broader peak with a flat top extending from 530 to $610 \mathrm{~nm} .{ }^{13}$ The PD spectrum was interpreted as reflecting overlapping contributions from the 6s-trans and 6s-cis configurations of RPSB with peak absorptions at 610 and $530 \mathrm{~nm}$, respectively. ${ }^{13}$ This explanation was supported by complete active space self-consistent field method (CASSCF) calculations that predict absorptions at 620 and $547 \mathrm{~nm}$ for the 6s-trans and 6s-cis forms, respectively, and also on the basis of separately obtained spectra of RPSB derivatives in which the $\beta$-ionone ring was chemically modified. 


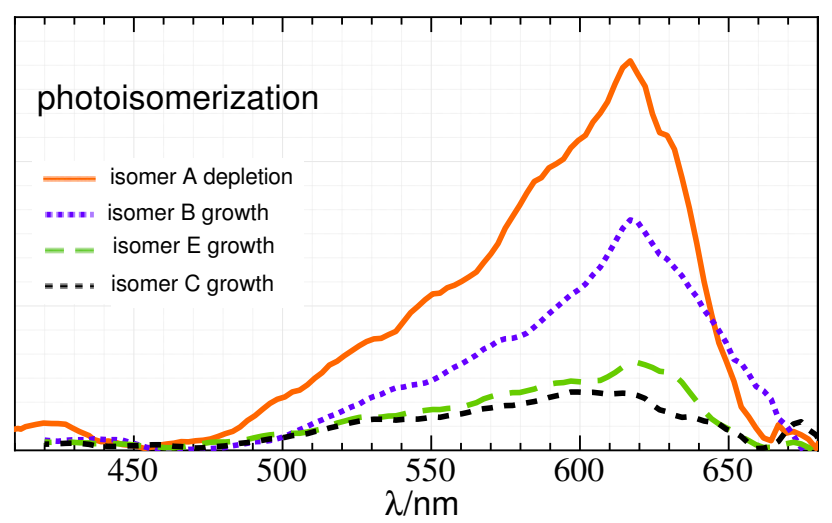

FIG. 5. PISA spectrum of RPSB in the gas phase normalised by photon flux. The raw spectrum and the laser power curve are available in the supplemental material (SM). ${ }^{20}$

The CASSCF methodology was subsequently criticised as lacking appropriate dynamical correlation by Valsson and Filippi, ${ }^{9}$ who, using an alternative multiconfigurational perturbative (NEVPT2) approach, predicted that the RPSB absorption wavelengths were 561 $\mathrm{nm}$ for the $6 \mathrm{~s}-$ trans, $537 \mathrm{~nm}$ for $6 \mathrm{~s}-$ cis(-), and $532 \mathrm{~nm}$ for $6 \mathrm{~s}-$ cis(+) configurations, a range of $29 \mathrm{~nm}$. The PISA spectrum, which does not exhibit a plateau but rather a well defined peak at $615 \mathrm{~nm}$, is consistent with the 6s-trans, 6s-cis(-) and 6s-cis(+) isomers having similar absorption wavelengths, near the experimental peak maximum (615 nm). This assumes, as predicted by our calculations and those of others, ${ }^{9,14}$ that the three $6 \mathrm{~s}$ isomers have similar energies and comparable populations at the effective temperature of the ions in the drift tube $(303 \mathrm{~K})$. It is worth noting that the experimental band maximum is very close to the wavelength $(619 \mathrm{~nm})$ predicted recently for the 6s-cis(-) isomer of RPSB (with an H atom rather than a butyl group bonded to the N atom) using the SF-BHHLYP/DH(d,p) and XMC-QDPT2/cc-pVDZ methods for the $S_{0}$ and $S_{1}$ states, respectively. ${ }^{21}$ Corresponding calculations for the $6 s-c i s(+)$ and $6 s-t r a n s$ isomers were not reported.

It is conceivable that some of the signal to shorter wavelength in the PISA spectrum (between 490 and $580 \mathrm{~nm}$ ) is due to different isomeric forms. However, it is likely that at least some of this signal comes from unresolved vibrational progressions that are excited due to changes in C-C bond lengths accompanying the $S_{1} \leftarrow S_{0}$ transition. ${ }^{9,21}$

In considering the differences between PD and PISA spectra it is first useful to examine why we observe photoisomerization of RPSB and not photodissociation to produce 
$m / z=248$ amu photofragments as detected previously. ${ }^{15}$ As explained in Section III A, photodissociation is negligible at the light intensities used in the present experiment $(<1 \%$ of the photoisomerization signal), although at much higher light intensities $(\times 10)$ we do observe $m / z=248$ amu photofragments. There are several possible explanations for the absence of PD in the drift cell at low light levels depending on whether the photon energy $\left(E_{p h}\right)$ is more or less than the energy of the dissociation barrier $\left(E_{d}\right)$.

1. A single visible photon provides sufficient energy to fragment the RPSB cation $\left(E_{p h}>E_{d}\right)$, however, collisional quenching in the PISA apparatus rapidly deprives the RPSB ions of energy so that they are unable to overcome the dissociation barrier yet are still able to surmount the much lower isomerization barriers. This would imply that the dissociation rate following single photon absorption is much lower than the collision rate $\left(5 \times 10^{8} \mathrm{~s}^{-1}\right)$. In this case, the PISA and PD spectra should be similar (assuming identical laser beam intensities, ion internal energies, and isomer distributions).

2. Absorption of a single visible photon does not provide most RSPB ions at $303 \mathrm{~K}$ with sufficient energy to overcome the dissociation barrier $\left(E_{p h}<E_{d}\right)$ but still gives them enough energy to photoisomerize. If this was true, previous PD studies of RPSB may have involved either hot ions and or multiphoton absorption, both of which can lead to spectral distortions. Regarding the possibility for multiple photon absorption, it is worth emphasising that measuring a linear dependence for the photodissociation signal against laser power over a limited intensity range does not necessarily exclude multiphoton absorption as the $S_{1} \leftarrow S_{0}$ transition of RPSB is strong and easily saturated at low light intensity. Beam intensities were not provided for previous PD spectroscopic studies of RPSB, ${ }^{10,11,13}$ making it impossible to compare them with those in the current PISA investigation $\left(1-2 \mathrm{~mJ} / \mathrm{cm}^{2} /\right.$ pulse).

Obviously, the magnitude of the dissociation barrier is a key issue in determining whether the PD spectrum will resemble the absorption spectrum. Assuming that single photon fragmentation occurs (Option 1 above - which at this stage we believe is not certain for cold RPSB ions), there are several possible explanations for the discrepancy between the PD and PISA spectra. For example, it is conceivable that the differences 
between the PD and PISA spectra are due to different isomeric compositions for the ion populations in the two experiments. Certainly, the presence of several isomer peaks in the ATD for electrosprayed RPSB cations (Figure 3) would be consistent with the PISA spectrum containing contributions from several low energy RPSB conformers, presumably including those in which the $\beta$-ionone ring is rotated around the $6 \mathrm{~s}$ bond. The fact that the peak in the PD spectrum is broader than the PISA spectrum might imply that an even larger range of isomers was present in the PD experiment.

It is also possible that the RPSB ions in the PD experiment are vibrationally hotter than those in the PISA experiment, leading to a broadening of the visible band in the PD spectrum. In the PISA experiment, the ions have undergone roughly $10^{6}$ collisions with $\mathrm{N}_{2}$ buffer gas molecules after exiting the first ion funnel and before being intercepted by the laser beam and therefore should be thermalised vibrationally at $T_{\text {eff }}=303 \mathrm{~K}$. The RPSB cations in the PD experiment, which were also created using an electrospray source, were presumably also thermalised in the source. However, conceivably they were heated through gas collisions while being stored or accelerated to $22 \mathrm{keV}$ to circulate in the ion storage ring. In principle, the effects of the creation, storage and acceleration process on the internal energy of the ions could be assessed by obtaining PD spectra of molecular ions that have well-resolved vibronic structure and which have previously been spectroscopically characterised under controlled conditions, including the para-dichlorobenzene cation, ${ }^{22}$ protonated tryptophan or tyrosine cations. ${ }^{23}$

The differences might also stem from a wavelength dependence for the photoisomerization yield. For example, it is conceivable that the pronounced $615 \mathrm{~nm}$ peak in the PISA spectrum of RPSB arises because the photoisomerization yield peaks near $615 \mathrm{~nm}$ and decreases for shorter wavelengths. The photoisomerization barriers $(40-130 \mathrm{~kJ} / \mathrm{mol}$; see Section IV) are much lower than the photon energies, so any variation of the photoisomerization yield with wavelength is unlikely to arise because of energy constraints. The evidence from condensed phase studies for a wavelength dependence of the photoisomerization yield is equivocal; for all-trans RPSB embedded in proteins and in acetonitrile solution, the photoisomerization quantum yield has been found to be independent of wavelength, whereas for all-trans RPSB in methanol the photoisomerization yield decreases by $25 \%$ going from 540 to $440 \mathrm{~nm}$ (although the variation is within the error range for the yield measurements). ${ }^{24}$ Although we cannot entirely discount the possibility for 
(a) Geometric Isomers

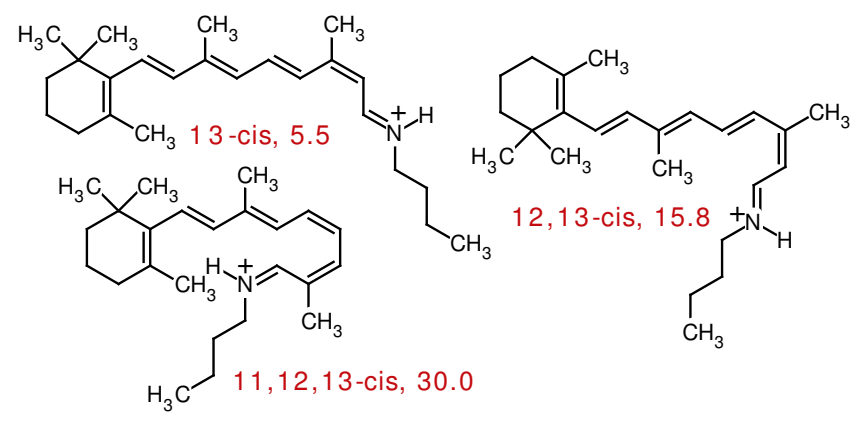

(b) Protomers
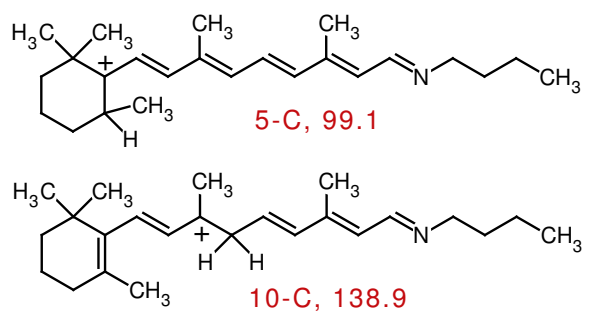

(c) Cyclic Isomers

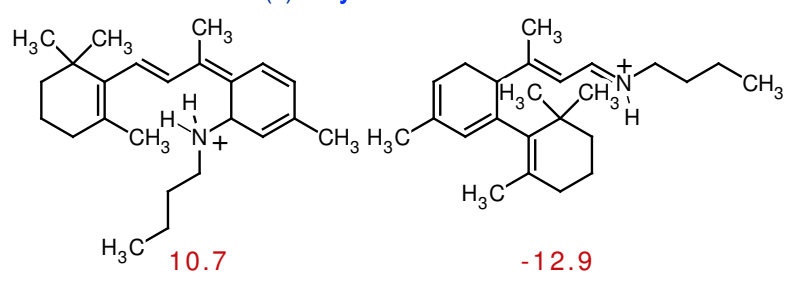

(d) Tricyclic Intermediate

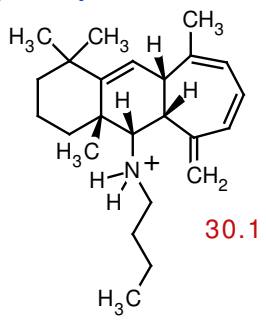

FIG. 6. Isomeric forms of RPSB determined from M06-2X/cc-pVDZ DFT calculations. In each case, the energy in $\mathrm{kJ} / \mathrm{mol}$ is given with respect to the all-trans isomer of RPSB. Energetic and structural details of these and other isomers are given in the SM. ${ }^{20}$ consider it unlikely. 


\section{ATD PEAK ASSIGNMENTS}

We now consider the RPSB isomers that are responsible for the observed ATD peaks. Although the conformational landscape of RPSB in the gas phase is extensive and complicated, the relative simplicity of the ATDs shown in Figure 3 which contain just 5 peaks suggests that only a restricted range of RPSB isomers are present in the IMS apparatus or that several isomers have very similar collision cross sections. As noted earlier, the most intense peak at $44.15 \mathrm{~ms}$ (peak A) most likely corresponds to all-trans RPSB, which has a predicted collision cross section of $220 \AA^{2}, 3.5 \%$ less than the measured value $\left(227.9 \AA^{2}\right)$. Assignment of the other peaks is less secure but they presumably belong to one of the three classes of molecules illustrated in Figure 6. 1. RPSB geometric isomers in which there are one or more cis bonds in the retinal chain (Figure 6a). 2. RPSB protomers, structures in which the excess proton is localised on a $\mathrm{C}$ atom rather than on the $\mathrm{N}$ atom (Figure 6b). 3. Cyclized versions of RPSB, akin to those previously observed in solution by Okamura et al. (Figure 6c). ${ }^{25}$ Below, we consider these explanations in turn.

To help assign the ATD peaks we employed M06-2X/cc-pVDZ DFT calculations to predict the equilibrium geometries, vibrational frequencies, energies, free energies of single, double and some triple cis geometrical isomers of RPSB, along with protomers, and cyclic forms. The M06-2X functional with its intermediate amount of exact exchange has been found to give an accurate description of RPSB's geometrical and energetic features. ${ }^{9}$ The geometrical structures were used to predict collision cross sections and mobilities using the classical trajectory method (TM) as instituted in the Mobcal program. ${ }^{26,27}$ Atomatom potential energy parameters for the interaction between $\mathrm{N}_{2}$ and the RPSB molecule were taken from ref. 28. Partial atomic charges were calculated using the Merz-SinghKollman scheme ${ }^{29}$ constrained to reproduce the molecular electrostatic potential. The DFT calculations were carried out using the Gaussian09 suite. ${ }^{30}$

\section{Geometric Isomers}

Perhaps the simplest and most likely explanation for the 5 observed ATD peaks is that they correspond to various geometric isomers of N-protonated RPSB of the type shown in Figure 6a. This explanation is consistent with the laser induced depletion of the 
all-trans isomer (Peak A) and enhancement of peaks B-E because in solution trans RPSB is known to photoisomerize to other cis isomers including 11-cis, 13-cis and 9-cis RPSB. ${ }^{31,32}$ These single cis RPSB isomers have similar absorption band profiles to the all-trans isomer but have $20-50 \%$ lower absorption cross sections..$^{31,32}$ Therefore, simultaneous irradiation of an ion packet consisting of various geometric isomers of RSPB can be expected to result in net conversion of all-trans RPSB isomer to other cis isomers.

Considering only the conjugated retinal chain and ignoring rotations about the $6 \mathrm{~s}$ bond and about the bonds in the butyl chain, there are 256 different trans-cis isomers. Here we have undertaken preliminary DFT explorations of the geometries and energies of $\sim 100$ different isomers, including single cis, double cis, and selected triple cis isomers of the retinal chain. We also calculated barriers for isomerization between the all-trans isomer to the single cis isomers and from single cis isomers to selected double cis isomers. Computed properties of the single cis isomers (averaged for internal rotation around the 6s bond, vide infra) are compiled in Table II. More comprehensive data for single cis, double cis and some triple cis isomers are provided in the SM. ${ }^{20}$

The single cis isomers are predicted to lie $6-24 \mathrm{~kJ} / \mathrm{mol}$ above the all-trans isomer and are possibly present in significant abundance in the drifting ion population. Barriers for transcis isomerization about the retinal bonds are relatively high and exhibit an alternation such that barriers for rotation about the formal double bonds are higher than for rotation about the single bonds, although the difference diminishes moving away from the $\beta$-ionone ring. Isomerization of the all-trans isomer to form the 7-cis, 9-cis, 11-cis and 13-cis isomers is calculated to require surmounting $100-130 \mathrm{~kJ} / \mathrm{mol}$ barriers on the $S_{0}$ surface whereas barriers for isomerization around the 8,10,12 and 14 single bonds range from 40 to $80 \mathrm{~kJ} / \mathrm{mol}$ (see Table II). One can expect that the 8-cis, 9-cis, 12-cis and 13-cis isomers, which all have energies $\leq 15 \mathrm{~kJ} / \mathrm{mol}$ above the ground state, will be formed from the trans isomer in the heated desolvation capillary where the temperature is $\sim 500 \mathrm{~K}$, and will probably survive the passage through the drift region.

It is important to recognise that isomers that have similar energies and are separated by low barriers will interconvert as they drift through the apparatus and so will generate a single ATD peak. For example, as previously noted, ${ }^{9}$ in the gas phase RPSB has 3 low-lying conformers corresponding to rotation around the $6 \mathrm{~s}$ bond, that have similar energies and relatively low interconversion barriers. DFT calculations by Valsson et al. at the M06-2X/cc- 
TABLE II. Data for low energy conformers of RPSB determined from M06-2X/cc-pVDZ DFT calculations. In each case the 6 s bond is in the (-) configuration (see Fig. 1). Included are zero-point corrected energies $(\Delta E)$ and free energies $(\Delta G$ at $T=298 \mathrm{~K})$ relative to that of the lowest energy isomer, barrier height for isomerization from the trans isomer, and average collision cross section $(\sigma)$ in $\mathrm{N}_{2}$ calculated using Eqn. 1. More complete calculated data for RPSB isomers are reported in the SM. ${ }^{20}$

\begin{tabular}{ccccc}
\hline \hline & $\Delta E$ & $\Delta G$ & barrier & $\sigma_{\text {calc }}$ \\
& $\mathrm{kJ} / \mathrm{mol}$ & $\mathrm{kJ} / \mathrm{mol}$ & $\mathrm{kJ} / \mathrm{mol}$ & $\AA^{2}$ \\
& & & & (calc) \\
\hline trans & 0 & 0 & & 219.9 \\
7-cis & 16.2 & 18.0 & 117 & 216.0 \\
8-cis & 11.5 & 10.8 & 42 & 218.4 \\
9-cis & 6.0 & 8.3 & 131 & 216.4 \\
10-cis & 23.6 & 27.1 & 60 & 217.9 \\
11-cis & 20.2 & 15.8 & 113 & 217.6 \\
12-cis & 10.7 & 14.2 & 68 & 220.3 \\
13-cis & 5.5 & 3.8 & 100 & 219.2 \\
14-cis & 17.9 & 19.8 & 81 & 219.0 \\
\hline
\end{tabular}

pVDZ level predict that the isomers are 6s-cis(-) with $\phi=-38.8^{\circ} \Delta E=0 \mathrm{~kJ} / \mathrm{mol}$, 6s-trans with $\phi=-170.1^{\circ} \Delta E=+0.5 \mathrm{~kJ} / \mathrm{mol}$, 6s-cis $(+)$ with $\phi=+41.8^{\circ} \Delta E=+1.4 \mathrm{~kJ} / \mathrm{mol} .{ }^{9}$ Energy barriers between the 6s-cis(-) and 6s-cis(+) forms and between 6s-cis(-) and trans forms are 6 and 14 $\mathrm{kJ} / \mathrm{mol}$, respectively. ${ }^{9}$ Energies and geometries from our M06-2X/cc-pVDZ calculations are very similar $\left(\right.$ see $\left.\mathrm{SM}^{20}\right)$. Under the conditions prevailing in the drift region $\left(T_{\text {eff }} \sim 303 \mathrm{~K}\right.$, collision rate $\sim 5 \times 10^{8} \mathrm{~s}^{-1}$ ), these $6 \mathrm{~s}$ isomers are expected to interconvert on timescales short compared to the drift time ( $\sim 45 \mathrm{~ms}$ ) and so should appear as a single peak in the ATD. Comparable DFT calculations show that the barriers for internal rotation around the 6s bond are also low for the 7-cis, 8-cis, 9-cis, 10-cis, 11-cis, 12-cis and 13-cis isomers, and that in each case the three $6 \mathrm{~s}$ conformers should interconvert as they traverse the drift region. To take this conformational averaging into account, we estimated the average 
collision cross-section $(\bar{\sigma})$ for interconverting population of $6 \mathrm{~s}$ isomers as,

$$
\bar{\sigma}=\sum_{i=1}^{3} \sigma_{i} e^{\frac{-\Delta G_{i}}{R T}} / \sum_{i=1}^{3} e^{\frac{-\Delta G_{i}}{R T}}
$$

where the sum is over the 6s-cis(-), 6s-cis(+) and 6s-trans isomers, and in each case $\Delta G_{i}$ is the relative free energy at $T=298 \mathrm{~K}$ (close to the effective temperature in the drift region). Averaged cross-sections for selected single cis isomers along with isomerization barriers are listed in Table II.

The n-butyl chain of RPSB is also expected to undergo conformational rearrangements as the RPSB ions drift through the buffer gas because typical barrier heights for rotation around the N-C and C-C bonds in the alkyl chain are expected to be $\leq 20 \mathrm{~kJ} / \mathrm{mol}$. At this stage, we have not taken these butyl chain rearrangements into account, restricting our attention to isomers in which the butyl chain is fully extended. Conformational rearrangements of the butyl chain should lead on average to somewhat more compact forms with slightly higher mobilities than the extended forms. Here we assume that the conformational averaging of the butyl chain has a similar effect on the drift mobilities for the different RPSB isomers. Eventually, estimating the effect of the conformational rearrangements of the butyl chain on the drift mobility is probably best accomplished using a molecular mechanics approach.

To explore whether the measured ATD is consistent with a thermal population of single, double and triple cis RPSB isomers, we have simulated the ATD using calculated energies and mobilities for $\sim 100$ different energetically low-lying geometric isomers with averaging over the $6 \mathrm{~s}$ rotation for the collision cross section. This was achieved by associating each isomer with a Gaussian peak $(\mathrm{fwhm}=0.6 \mathrm{~ms})$ centred at the predicted arrival time (determined from its calculated mobility), and an intensity that assumes a Boltzmann distribution of isomers at $T=600 \mathrm{~K}$ ( $100 \mathrm{~K}$ higher than the temperature of the electrospray desolvation capillary). The collision cross sections for the different isomers were scaled such that the predicted arrival time for the all-trans isomer corresponded to the measured arrival time for peak A. The simulation assumes that the ions rearrange relatively slowly over the barriers connecting the various geometric isomeric forms, which typically have heights of $\sim 100 \mathrm{~kJ} / \mathrm{mol}$ for rotation around the odd C-C bonds of the retinal chain and $\sim 50 \mathrm{~kJ} / \mathrm{mol}$ for rotation around the even bonds (Table II).

Notwithstanding the crudity of the underlying assumptions, the simulated ATD shown 
in Figure 3c reproduces two fastest peaks in the ATD (peaks A and B) reasonably well. Several single cis isomers (8-cis, 12-cis, 13-cis) are predicted to have a similar collision crosssections to the all-trans isomer and lie sufficiently low in energy $(\leq 15 \mathrm{~kJ} / \mathrm{mol}$ with respect to the all-trans isomer) to also contribute to the dominant peak A. The slightly earlier peak B, centered at $43.25 \mathrm{~ms}$ corresponds to ions with $2 \%$ smaller collision cross section, and can be assigned primarily to the 9-cis form with contributions from the 7-cis and 11-cis forms (predicted to lie 6.6, $16.5 \& 21.3 \mathrm{~kJ} / \mathrm{mol}$ above the all-trans form, respectively). A simulated laser on/off ATD generated by assuming that the laser pulse merely heats the molecules from 600 to $800 \mathrm{~K}$ is shown in Figure 3c. The resemblance to the measured laser on/off ATD is also reasonable in the region associated with the single and double cis isomers (peaks A, B and E).

Generally, the simulated ATD is less satisfactory in the region near the earlier peaks C and D (arrival times 41.30 and $40.10 \mathrm{~ms}$ ), which correspond to ions with collision cross sections that are respectively 7 and $9 \%$ smaller than the all-trans isomer, and must be due to more compact isomers. Calculated collision cross sections based on DFT structures suggest that these earlier peaks are possibly associated with geometric isomers possessing three adjacent cis bonds (e.g. 9-cis 10-cis 11-cis , 11-cis 12-cis 13-cis or 12-cis 13-cis 14cis isomers). These triple-cis isomers lie $\geq 30 \mathrm{~kJ} / \mathrm{mol}$ above the ground state and are predicted to have very low populations at $T=600 \mathrm{~K}$ but become significantly populated at higher temperatures and may become important following photoexcitation.

\section{Protomers}

As noted above, an alternative explanation for the faster isomers (peaks $\mathrm{C}$ and $\mathrm{D}$ and perhaps peak E) is that they correspond to RPSB protomers, structures in which the excess proton is localised on a C atom rather than on the $\mathrm{N}$ atom (Figure 6b). DFT calculations demonstrate that these protomer structures lie $79-191 \mathrm{~kJ} / \mathrm{mol}$ above the all-trans form of RPSB and therefore they are less likely to be formed in the electrospray ion source than the trans-cis geometric isomers (see $\mathrm{SM}^{20}$ for protomer structures and energies ). Preliminary DFT and mobility calculations suggest that the protomers in their extended configurations have mobilities that are comparable to the geometric isomers of RPSB and have predicted arrival times ranging from 42.7 to $44.5 \mathrm{~ms}$. The lowest energy protomer is predicted to 
have the excess proton attached to the $14 \mathrm{C}$, lie $79 \mathrm{~kJ} / \mathrm{mol}$ above the $\mathrm{N}$ protonated form, and have an arrival time of $43.7 \mathrm{~ms}$. Intriguingly, if the peaks $\mathrm{C}$ and $\mathrm{E}$ indeed correspond to protomers, then one would be obliged to conclude that visible photo-excitation can induce intramolecular proton transfer in RPSB. Whether this process is feasible depends of course on the magnitude of the energy barrier separating them from the $\mathrm{N}$ protonated version.

\section{Cyclic versions of RPSB}

A third explanation for the faster, more compact isomers (peaks $C$ and $D$ ) is that they are due to cyclic versions of RPSB similar to those previously observed by Okamura et al. ${ }^{25}$ (Figure 6c). Generally, these proposed cyclic versions, two examples of which are illustrated in Figure $6 c$, have collision cross sections that are 10-15\% less than all-trans RPSB and are predicted to arrive in the 35-41 ms range. For example, the lowest energy cyclic form we have managed to locate computationally is shown on the right of Figure $6 \mathrm{c}$ and lies $12.9 \mathrm{~kJ} / \mathrm{mol}$ below the all-trans form of RPSB. Its predicted collision cross section is $\sim 10 \%$ less than the all-trans RPSB isomer and its predicted arrival time is $39.6 \mathrm{~ms}$, slightly less than peak D. Again, assignment of peaks C and D to cyclic structures would imply that RPSB can be photochemically transformed into cyclic versions of RPSB in the gas phase.

We also considered the tricyclic structure shown in Figure 6d, which has been proposed as an intermediate in the photodissociation of RPSB under collision free conditions. ${ }^{15}$ It has been postulated that the tricyclic isomer forms rapidly from RPSB following excitation of the $S_{1} \leftarrow S_{0}$ transition and then decomposes slowly (on the ms timescale) to give a charged bicyclic fragment with $m / z=248$ amu and a toluene molecule (or isomer thereof). The most likely stereoisomer of the tricyclic structure is calculated to have an energy $30 \mathrm{~kJ} / \mathrm{mol}$ above all-trans RPSB and a collision cross section with $\mathrm{N}_{2}$ of $188 \AA^{2}$, around $15 \%$ lower than that of the all-trans isomer. Its predicted arrival time is $38 \mathrm{~ms}$, well before any of the observed ATD peaks. Therefore, this tricylic intermediate appears unlikely to be formed, either through collisions or photo excitation, under the higher pressure conditions prevailing in our apparatus.

In principle, the absorption spectra of the isomers can be used as a means for their 
identification, deciding whether they are indeed due to geometric RPSB isomers (Figure 6a) or to various protomers and cyclic versions (Figures $6 \mathrm{~b} \& \mathrm{c}$ ). These latter species possess shorter conjugated sequences than N-protonated RPSB and will therefore have distinctly different absorption bands, most likely occurring to shorter wavelength. As shown in Section III B below, there is no indication that the different isomers (peaks A-E) have significantly different absorption profiles, although admittedly we have not yet conducted systematic scans at wavelengths shorter than $410 \mathrm{~nm}$.

\section{PHOTOISOMERIZATION MECHANISM}

As far as we are aware this is the first experimental work to address photoisomerization of RPSB in the gas phase. In solution and when embedded in a protein, trans RPSB is hypothesised to photoisomerize to cis isomers (e.g. 11-cis, 13-cis) through a conical intersection (CI) linking the $\mathrm{S}_{1}$ and $\mathrm{S}_{0}$ surfaces. ${ }^{33}$ Data on the photoisomerization pathways of RPSB in methanol solution are summarised in Table III. The photoisomerization for all-trans RPSB in methanol has been found to proceed with high specificity and has a measured quantum yield of $0.17 \pm 0.03 .{ }^{32}$ HPLC analysis of photoproducts shows that trans RPSB in methanol photoisomerizes primarily to 11-cis RPSB with formation of 13-cis, 9-cis, and 7-cis isomers being less efficient by an order of magnitude. ${ }^{32}$ On the other hand, both the 11-cis and 13-cis forms thermally rearrange primarily to give all-trans RPSB. ${ }^{32}$

Is there a similar specificity in the gas phase? Does photoisomerization proceed through a CI, driving isomerization around, for example, the 11,12 bond to form the 11-cis isomer (direct photochemical mechanism), or is it essentially a statistical process, that follows internal conversion (IC) with $\sim 200 \mathrm{~kJ} / \mathrm{mol}$ of electronic energy transformed into vibrational energy and subsequent statistical exploration of the isomeric landscape? Recent theoretical studies predict that the photo-excited RPSB molecule in the gas phase is rather floppy and that there are multiple relaxation paths as the molecule moves away from the FC region (with rotations around formal double and single bonds); some of these paths move towards CIs whereas others do not. ${ }^{34}$ Ultimately, the fate of molecules passing through a CI linking the $S_{1}$ and $S_{0}$ surfaces may be the same as for molecules that undergo IC. This is because collisions are much less frequent in the gas phase than in solution and there is no effective means for dissipating vibrational energy liberated during passage from the $S_{1} F C$ 
TABLE III. Photophysical data for all-trans, 13-cis, 11-cis and 9-cis RPSB dissolved in methanol taken from ref. 32. Included are wavelengths of absorption maxima $\left(\lambda_{\max }\right)$, extinction coefficients $(\epsilon)$, photoisomerization quantum yields $\left(\phi_{i s o}\right)$, and main photoisomerization products.

\begin{tabular}{|c|c|c|c|c|}
\hline & $\begin{array}{c}\lambda_{\max } \\
\mathrm{nm}\end{array}$ & $\begin{array}{c}\epsilon \\
\mathrm{M}^{-1} \mathrm{~cm}^{-}\end{array}$ & $\phi_{i s o}$ & products \\
\hline trans & 445 & 51800 & $0.17 \pm 0.03$ & $9 \mathrm{c}), 0.14(11 \mathrm{c}), 0.01(13 \mathrm{c})$ \\
\hline 9-cis & 432 & 39400 & $0.13 \pm 0.03$ & $0.07(\mathrm{t}), 0.06(11 \mathrm{c})$ \\
\hline $11-c i s$ & 442 & 23300 & $0.22 \pm 0.05$ & $0.22(\mathrm{t})$ \\
\hline $13-c i s$ & 434 & 23900 & $0.13 \pm 0.02$ & $0.11(t), 0.01(11 c)$ \\
\hline
\end{tabular}

region, down through the $\mathrm{CI}$, and then on the $\mathrm{S}_{0}$ surface. Under these circumstances, even if the system reaches a particular isomeric form on the $\mathrm{S}_{0}$ surface it possesses sufficient vibrational energy to rearrange to other forms. Eventually, collisions with buffer gas $\left(k_{c} \sim 5 \times 10^{8} \mathrm{~s}^{-1}\right)$ cool the system below the isomerization barriers $\left(V_{b} \sim 100 \mathrm{~kJ} / \mathrm{mol}\right)$. This statistical picture of photoisomerization in the gas phase is supported by the reasonable agreement between observed and predicted laser on/off difference ATDs if we presume that the laser pulse merely heats the ion population from 600 to $800 \mathrm{~K}$ (Figure 3c).

Previously, we used a statistical approach to explain the photoisomerization behaviour of the polymethine dye $\mathrm{DTC}^{+}$, which consists of two aromatic groups connected by 4 conjugated C-C bonds, and which we have previously studied using PISA spectroscopy. ${ }^{17}$ For $\mathrm{DTC}^{+}$, a time-dependent master equation simulation successfully predicted the preferential formation of the all-trans and 8,9-cis isomers following laser excitation and the absence of the 2,8-cis form. Simulations of the relaxation on the ground state electronic surface demonstrated that following IC, the $\mathrm{DTC}^{+}$molecules explore the conformational landscape as they slowly cool through buffer gas collisions. In line with experimental observations, the simulations showed that the 8,9-cis isomer is preferred over the 2,8-cis isomer because it lies lower in energy (by $\sim 5 \mathrm{~kJ} / \mathrm{mol}$ ) and because it is separated from the trans isomer by a substantially higher barrier ( 89.7 versus $43.6 \mathrm{~kJ} / \mathrm{mol})$. Because of rapid 
isomeric interconversion, the preference for the more stable 8,9-cis isomer was predicted to persist even when it was assumed that the 2,8-cis isomer was initially populated by passage through a CI. RPSB is a significantly more complicated system than $\mathrm{DTC}^{+}$and we have not yet modelled its isomerization behaviour using the master equation approach. However, as for $\mathrm{DTC}^{+}$, IC with subsequent slow collisional relaxation should favour energetically low-lying isomers that are separated from other forms by large isomerization barriers.

\section{CONCLUSIONS}

Protonated retinal Schiff base ions have been formed using electrospray ionisation and introduced into a ion mobility spectrometer where they are irradiated with tuneable visible radiation. The RPSB ion population is dominated by the all-trans geometric isomer but also contains several more compact isomeric forms that can also be formed through photo excitation of the all-trans isomer. The photoisomerization action spectrum of RPSB exhibits a single, well-defined $S_{1} \leftarrow S_{0}$ peak at $615 \pm 5 \mathrm{~nm}$. The band differs in shape from the recently reported photodissociation spectrum which displays a broad band with a flat top that extends from 530 to $610 \mathrm{~nm} .{ }^{13}$ The differences between the PISA and PD spectra are suggested to arise either from differences in the wavelength dependence for the photodissociation yield or photoisomerization yield, differences in the internal energy of the ion populations, or presence of different isomers in the probed populations. Notably, the PISA data suggests that the $S_{1} \leftarrow S_{0}$ absorption wavelengths of the 6s-cis(-), 6s-cis(+) and 6s-trans forms of RPSB are similar.

There are several obvious extensions to the current work. First, it would be useful to prepare and characterise other RPSB isomers (9-cis , 11-cis , 13-cis isomers), measuring their collision cross sections and PISA spectra so that the data can be compared with the all-trans RPSB results presented here. This would hopefully help assign the ion mobility peaks observed in this study. Second, it would useful to subject the RPSB ions to an IMS step before laser irradiation so that a particular isomer population can be irradiated cleanly without interference from other isomers. This would also help in assigning the ATD peaks and would allow one to determine whether other RPSB protomers or cyclized 
versions are present in the drifting ion population as they should have distinct absorption spectra. From a theoretical perspective, reliable calculations for the dissociation barrier would enable us understand whether photodissociation of cold RPSB is a single or multi photon process and perhaps help identify the source of differences between the PISA and PD spectra.

\section{ACKNOWLEDGMENTS}

This research was supported under the Australian Research Council's Discovery Project funding scheme (Project Numbers DP110100312 and DP120100100). We acknowledge Catrin Goeschen and Luke Gamon for helping with the RPSB synthesis and HPLC characterisation. We thank Professor Matthew Bush for providing a modified version of the Mobcal code with $\mathrm{N}_{2}$ collision parameters described in ref. 28 .

\section{REFERENCES}

${ }^{1}$ P. Hamm, M. Zurek, T. Röschinger, H. Patzelt, D. Oesterhelt, and W. Zinth, Chem. Phys. Lett. 263, 613 (1996).

${ }^{2}$ O. P. Ernst, D. T. Lodowski, M. Elstner, P. Hegemann, L. S. Brown, and H. Kandori, Chem. Rev 114, 126 (2013).

${ }^{3}$ J. Herbst, K. Heyne, and R. Diller, Science 297, 822 (2002).

${ }^{4}$ R. Govindjee, S. P. Balashov, and T. G. Ebrey, Biophys. J. 58, 597 (1990).

${ }^{5}$ O. Valsson, P. Campomanes, and I. Tavernelli, J. Chem. Theory Comput. 9, 2441 (2013).

${ }^{6}$ W. Wang, Z. Nossoni, T. Berbasova, C. T. Watson, I. Yapici, K. S. S. Lee, C. Vasileiou, J. H. Geiger, and B. Borhan, Science 338, 1340 (2012).

${ }^{7}$ T. Sovdat, G. Bassolino, M. Liebel, C. Schnedermann, S. P. Fletcher, and P. Kukura, J. Am. Chem. Soc. 134, 8318 (2012).

${ }^{8}$ G. Bassolino, T. Sovdat, M. Liebel, C. Schnedermann, B. Odell, T. D. W. Claridge, P. Kukura, and S. P. Fletcher, J. Am. Chem. Soc. 136, 2650 (2014).

${ }^{9}$ O. Valsson and C. Filippi, J. Phys. Chem. Lett. 3, 908 (2012).

${ }^{10}$ L. H. Andersen, I. B. Nielsen, M. B. Kristensen, M. O. A. El Ghazaly, S. Haacke, M. B. Nielsen, and M. Å. Petersen, J. Am. Chem. Soc. 127, 12347 (2005). 
${ }^{11}$ I. Nielsen, L. Lammich, and L. Andersen, Phys. Rev. Lett. 96, 018304 (2006).

${ }^{12}$ L. Lammich, I. B. Nielsen, H. Sand, A. Svendsen, and L. H. Andersen, J. Phys. Chem. A 111, 4567 (2007).

${ }^{13}$ J. Rajput, D. B. Rahbek, L. H. Andersen, A. Hirshfeld, M. Sheves, P. Altoè, G. Orlandi, and M. Garavelli, Angew. Chem. Int. Ed. 49, 1790 (2010).

${ }^{14}$ Y. Toker, A. Svendsen, A. V. Bochenkova, and L. H. Andersen, Angew. Chem. Int. Ed. 51, 8757 (2012)

${ }^{15}$ L. Andersen, Y. Toker, H. Kiefer, J. Rajput, S. B. Nielsen, A. Bochenkova, P. Dugourd, R. Antoine, and D. B. Rahbek, Phys. Chem. Chem. Phys. 15, 19566 (2013).

${ }^{16}$ B. D. Adamson, N. J. A. Coughlan, R. Continetti, and E. J. Bieske, Phys. Chem. Chem. Phys. 15, 9540 (2013).

${ }^{17}$ B. D. Adamson, N. J. A. Coughlan, G. da Silva, and E. J. Bieske, J. Phys. Chem. A 117, 13319 (2013).

${ }^{18}$ H. E. Revercomb and E. A. Mason, Anal. Chem. 47, 970 (1975).

${ }^{19}$ A. K. Singh and R. V. Aruna, Biochimica et Biophysica Acta 1245, 167 (1995).

20“'See supplemental material at [url will be inserted by aip] for calculated data of RPSB isomers and for raw PISA spectra of RPSB,".

${ }^{21}$ P. Zhou, J. Liu, K. Han, and G. He, J. Comput. Chem. 35, 109 (2014).

${ }^{22}$ A. Dzhonson, D. Gerlich, E. J. Bieske, and J. P. Maier, J. Mol. Struct. 795, 93 (2006).

${ }^{23}$ T. R. Rizzo, J. A. Stearns, and O. V. Boyarkin, Int. Rev. Phys. Chem. 28, 481 (2009).

${ }^{24}$ G. Zgrablić, M. Ricci, A. M. Novello, and F. Parmigiani, Photochem. Photobiol. 86, 507 (2010).

${ }^{25}$ W. H. Okamura, A. R. De Lera, and W. Reischl, J. Am. Chem. Soc. 110, 4462 (1988).

${ }^{26}$ A. A. Shvartsburg and M. F. Jarrold, Chem. Phys. Letts. 261, 86 (1996).

${ }^{27}$ M. Mesleh, J. Hunter, A. Shvartsburg, G. Schatz, and M. Jarrold, J. Phys. Chem. 100, 16082 (1996).

${ }^{28}$ I. Campuzano, M. F. Bush, C. V. Robinson, C. Beaumont, K. Richardson, H. Kim, and H. I. Kim, Anal. Chem 84, 1026 (2012).

${ }^{29}$ U. C. Singh and P. A. Kollman, J. Comp. Chem. 5, 129 (1984).

${ }^{30}$ M. J. Frisch, G. W. Trucks, H. B. Schlegel, G. E. Scuseria, M. A. Robb, J. R. Cheeseman, G. Scalmani, V. Barone, B. Mennucci, G. A. Petersson, H. Nakatsuji, M. Caricato, X. Li, H. P. Hratchian, A. F. Izmaylov, J. Bloino, G. Zheng, J. L. Sonnenberg, M. Hada, 
M. Ehara, K. Toyota, R. Fukuda, J. Hasegawa, M. Ishida, T. Nakajima, Y. Honda, O. Kitao, H. Nakai, T. Vreven, J. A. Montgomery, Jr., J. E. Peralta, F. Ogliaro, M. Bearpark, J. J. Heyd, E. Brothers, K. N. Kudin, V. N. Staroverov, R. Kobayashi, J. Normand, K. Raghavachari, A. Rendell, J. C. Burant, S. S. Iyengar, J. Tomasi, M. Cossi, N. Rega, J. M. Millam, M. Klene, J. E. Knox, J. B. Cross, V. Bakken, C. Adamo, J. Jaramillo, R. Gomperts, R. E. Stratmann, O. Yazyev, A. J. Austin, R. Cammi, C. Pomelli, J. W. Ochterski, R. L. Martin, K. Morokuma, V. G. Zakrzewski, G. A. Voth, P. Salvador, J. J. Dannenberg, S. Dapprich, A. D. Daniels, Ö. Farkas, J. B. Foresman, J. V. Ortiz, J. Cioslowski, and D. J. Fox, “Gaussian 09 Revision A.1," Gaussian Inc. Wallingford CT 2009.

${ }^{31}$ K. A. Freedman and R. S. Becker, J. Am. Chem. Soc. 108, 1245 (1986).

${ }^{32}$ Y. Koyama, K. Kubo, M. Komori, H. Yasuda, and Y. Mukai, Photochem. Photobiol. 54, 433 (1991).

${ }^{33}$ D. Polli, P. Altoè, O. Weingart, K. M. Spillane, C. Manzoni, D. Brida, G. Tomasello, G. Orlandi, P. Kukura, R. A. Mathies, M. Garavelli, and G. Cerullo, Nature 467, 440 (2010).

${ }^{34}$ O. Valsson and C. Filippi, J. Chem. Theory Comput. 6, 1275 (2010).

${ }^{35}$ P. Hamm, M. Zurek, T. Röschinger, H. Patzelt, D. Oesterhelt, and W. Zinth, Chem. Phys. Lett. 263, 613 (1996).

${ }^{36}$ O. P. Ernst, D. T. Lodowski, M. Elstner, P. Hegemann, L. S. Brown, and H. Kandori, Chem. Rev 114, 126 (2013).

${ }^{37}$ J. Herbst, K. Heyne, and R. Diller, Science 297, 822 (2002).

${ }^{38}$ R. Govindjee, S. P. Balashov, and T. G. Ebrey, Biophys. J. 58, 597 (1990).

${ }^{39}$ O. Valsson, P. Campomanes, and I. Tavernelli, J. Chem. Theory Comput. 9, 2441 (2013).

${ }^{40}$ W. Wang, Z. Nossoni, T. Berbasova, C. T. Watson, I. Yapici, K. S. S. Lee, C. Vasileiou, J. H. Geiger, and B. Borhan, Science 338, 1340 (2012).

${ }^{41}$ T. Sovdat, G. Bassolino, M. Liebel, C. Schnedermann, S. P. Fletcher, and P. Kukura, J. Am. Chem. Soc. 134, 8318 (2012).

${ }^{42}$ G. Bassolino, T. Sovdat, M. Liebel, C. Schnedermann, B. Odell, T. D. W. Claridge, P. Kukura, and S. P. Fletcher, J. Am. Chem. Soc. 136, 2650 (2014).

${ }^{43}$ O. Valsson and C. Filippi, J. Phys. Chem. Lett. 3, 908 (2012).

${ }^{44}$ L. H. Andersen, I. B. Nielsen, M. B. Kristensen, M. O. A. El Ghazaly, S. Haacke, M. B. Nielsen, and M. A. Petersen, J. Am. Chem. Soc. 127, 12347 (2005). 
${ }^{45}$ I. Nielsen, L. Lammich, and L. Andersen, Phys. Rev. Lett. 96, 018304 (2006).

${ }^{46}$ L. Lammich, I. B. Nielsen, H. Sand, A. Svendsen, and L. H. Andersen, J. Phys. Chem. A 111, 4567 (2007).

${ }^{47}$ J. Rajput, D. B. Rahbek, L. H. Andersen, A. Hirshfeld, M. Sheves, P. Altoè, G. Orlandi, and M. Garavelli, Angew. Chem. Int. Ed. 49, 1790 (2010).

${ }^{48}$ Y. Toker, A. Svendsen, A. V. Bochenkova, and L. H. Andersen, Angew. Chem. Int. Ed. 51, 8757 (2012).

${ }^{49}$ L. Andersen, Y. Toker, H. Kiefer, J. Rajput, S. B. Nielsen, A. Bochenkova, P. Dugourd, R. Antoine, and D. B. Rahbek, Phys. Chem. Chem. Phys. 15, 19566 (2013).

${ }^{50}$ B. D. Adamson, N. J. A. Coughlan, R. Continetti, and E. J. Bieske, Phys. Chem. Chem. Phys. 15, 9540 (2013).

${ }^{51}$ B. D. Adamson, N. J. A. Coughlan, G. da Silva, and E. J. Bieske, J. Phys. Chem. A 117, 13319 (2013).

${ }^{52}$ H. E. Revercomb and E. A. Mason, Anal. Chem. 47, 970 (1975).

${ }^{53}$ A. K. Singh and R. V. Aruna, Biochimica et Biophysica Acta 1245, 167 (1995).

54“See supplemental material at [url will be inserted by aip] for calculated data of RPSB isomers and for raw PISA spectra of RPSB,".

${ }^{55}$ P. Zhou, J. Liu, K. Han, and G. He, J. Comput. Chem. 35, 109 (2014).

${ }^{56}$ A. Dzhonson, D. Gerlich, E. J. Bieske, and J. P. Maier, J. Mol. Struct. 795, 93 (2006).

${ }^{57}$ T. R. Rizzo, J. A. Stearns, and O. V. Boyarkin, Int. Rev. Phys. Chem. 28, 481 (2009).

${ }^{58}$ G. Zgrablić, M. Ricci, A. M. Novello, and F. Parmigiani, Photochem. Photobiol. 86, 507 (2010).

${ }^{59}$ W. H. Okamura, A. R. De Lera, and W. Reischl, J. Am. Chem. Soc. 110, 4462 (1988).

${ }^{60}$ A. A. Shvartsburg and M. F. Jarrold, Chem. Phys. Letts. 261, 86 (1996).

${ }^{61}$ M. Mesleh, J. Hunter, A. Shvartsburg, G. Schatz, and M. Jarrold, J. Phys. Chem. 100, 16082 (1996).

${ }^{62}$ I. Campuzano, M. F. Bush, C. V. Robinson, C. Beaumont, K. Richardson, H. Kim, and H. I. Kim, Anal. Chem 84, 1026 (2012).

${ }^{63}$ U. C. Singh and P. A. Kollman, J. Comp. Chem. 5, 129 (1984).

${ }^{64}$ M. J. Frisch, G. W. Trucks, H. B. Schlegel, G. E. Scuseria, M. A. Robb, J. R. Cheeseman, G. Scalmani, V. Barone, B. Mennucci, G. A. Petersson, H. Nakatsuji, M. Caricato, X. Li, H. P. Hratchian, A. F. Izmaylov, J. Bloino, G. Zheng, J. L. Sonnenberg, M. Hada, 
M. Ehara, K. Toyota, R. Fukuda, J. Hasegawa, M. Ishida, T. Nakajima, Y. Honda, O. Kitao, H. Nakai, T. Vreven, J. A. Montgomery, Jr., J. E. Peralta, F. Ogliaro, M. Bearpark, J. J. Heyd, E. Brothers, K. N. Kudin, V. N. Staroverov, R. Kobayashi, J. Normand, K. Raghavachari, A. Rendell, J. C. Burant, S. S. Iyengar, J. Tomasi, M. Cossi, N. Rega, J. M. Millam, M. Klene, J. E. Knox, J. B. Cross, V. Bakken, C. Adamo, J. Jaramillo, R. Gomperts, R. E. Stratmann, O. Yazyev, A. J. Austin, R. Cammi, C. Pomelli, J. W. Ochterski, R. L. Martin, K. Morokuma, V. G. Zakrzewski, G. A. Voth, P. Salvador, J. J. Dannenberg, S. Dapprich, A. D. Daniels, Ö. Farkas, J. B. Foresman, J. V. Ortiz, J. Cioslowski, and D. J. Fox, “Gaussian 09 Revision A.1," Gaussian Inc. Wallingford CT 2009.

${ }^{65}$ K. A. Freedman and R. S. Becker, J. Am. Chem. Soc. 108, 1245 (1986).

${ }^{66}$ Y. Koyama, K. Kubo, M. Komori, H. Yasuda, and Y. Mukai, Photochem. Photobiol. 54, 433 (1991).

${ }^{67}$ D. Polli, P. Altoè, O. Weingart, K. M. Spillane, C. Manzoni, D. Brida, G. Tomasello, G. Orlandi, P. Kukura, R. A. Mathies, M. Garavelli, and G. Cerullo, Nature 467, 440 (2010).

${ }^{68}$ O. Valsson and C. Filippi, J. Chem. Theory Comput. 6, 1275 (2010). 